\title{
Estimation of momentum and heat fluxes using dissipation and flux-variance methods in the unstable surface layer
}

\author{
Cheng-I Hsieh and Gabriel G. Katul \\ School of the Environment, Duke University, Durham, North Carolina \\ John Schieldge \\ Jet Propulsion Laboratory, California Institute of Technology, Pasadena \\ John Sigmon and Kenneth R. Knoerr \\ School of the Environment, Duke University, Durham, North Carolina
}

\begin{abstract}
Dissipation and flux-variance methods, derived from the turbulent kinetic energy and temperature variance budget equations in conjunction with Monin-Obukov similarity theory, were used to estimate surface fluxes of momentum and sensible heat. To examine the performance of these two methods, direct eddy correlation measurements were carried out above a nonuniform grass-covered forest clearing in Durham, North Carolina. The dissipation method sensible heat flux predictions were in good agreement with eddy correlation measurements. Also, the flux-variance method reproduced the measured sensible heat flux well following an adjustment to the similarity constant. However, the momentum flux (or friction velocity) estimated by the dissipation and fluxvariance methods were both inferior to those for sensible heat flux. The data from this experiment indicated that the above two methods are sensitive to the dimensionless wind shear $\left(\phi_{m}\right)$ and temperature standard deviation $\left(\phi_{\theta}\right)$ functions. On the basis of dimensional analysis and the temperature variance budget equation a new dissipation approach for estimating sensible heat flux was derived. The similarity constant for this new approach was shown to be around 1.6 for uniform surfaces and from the data of this experiment.
\end{abstract}

\section{Introduction}

The turbulent momentum $\left(\rho \mu_{*}{ }^{2}\right)$ and sensible heat $(H)$ fluxes are two of the important parameters necessary to quantify land-atmosphere interactions and energy transport processes in the atmospheric surface layer (ASL). On the basis of turbulence measurements in the ASL, several methods have been developed to determine these fluxes, including (1) the direct eddy correlation method, (2) the dissipation method, and (3) the flux-variance method.

The eddy correlation method, considered to be the most accurate method, measures the fluxes directly by measuring the covariances between the turbulent flow variables. However, routine application of this method in field experiments requires (1) high sampling rates (e.g., 10-20 Hz), (2) precise orientation and alignment of sensors, and (3) long sampling duration (e.g., $30 \mathrm{~min}$ ) to obtain stable statistics [see Brutsaert, 1982, pp. 191; Champagne et al., 1977].

The dissipation method (variance budget method) was first introduced by Deacon [1959] and evaluated by Taylor [1961]. This method is based on the budget equations of turbulent kinetic energy (TKE) and temperature variance in conjunction with Monin and Obukhov [1954] similarity theory (hereafter referred to as MOST). In this method the mean dissipation rates of TKE and temperature variance from high-frequency

Copyright 1996 by the American Geophysical Union.

Paper number 96WR01337. 0043-1397/96/96WR-01337\$09.00 (e.g., $>20 \mathrm{~Hz}$ ) velocity and temperature measurements are used to estimate momentum and heat fluxes. Although the method requires high-frequency measurements, it is not as sensitive to the sensors alignment and orientation as the eddy correlation method. The method proved to be well suited for flux measurements above the ocean. Many investigators [e.g., Kader, 1992; Dyer and Hicks, 1982; Champagne et al., 1977] have reported that the dissipation method agreed well with the measurements.

The flux-variance method (temperature fluctuation method) is based on MOST for the dimensionless function of temperature standard deviation $\left(\phi_{\theta}\right)$. This method uses the fluxvariance relations, derived above uniform flat terrain, to estimate the momentum and heat fluxes. Once the fluctuations of temperature and vertical wind speed or mean horizontal wind speed are known, the sensible heat flux and friction velocity $\left(u_{*}\right)$ can be estimated iteratively. After Tillman [1972] published his first paper on the flux-variance approach, many investigators evaluated this method for a wide range of surface types and stability conditions and concluded good to fair performance [see Katul et al., 1995; Albertson et al., 1995; Kustas et al., 1994; de Bruin, 1994; de Bruin et al., 1993, 1991; Padro, 1993; Padro et al., 1992; Lloyd et al., 1991; Weaver, 1990; Wesely, 1988]. However, it is evident that the accuracy of this method depends on the validity of $\phi_{\theta}$. Katul et al. [1995] found that the flux-variance method is less sensitive to the ground heating nonuniformity for near convective conditions, but it is sensitive for dynamic and dynamic-convective conditions. Weaver [1990] suggested that if the flux is small or the surface is heteroge- 
neous, it is necessary to determine a local flux-variance relation (i.e., to adjust the flux-variance relation with land use type) prior to applying this method.

It is the nature of these limitations and the importance of developing and testing simple methods to determine $H$ and $u_{*}$ that motivated this study. The objectives of this study are to compare the dissipation and flux-variance methods with eddy correlation measurements above a nonuniform grass-covered forest and asses the sensitivity of these two methods to site nonuniformity. Also, using dimensional analysis, a new dissipation approach was proposed for estimating $H$. For this purpose, 56- and $21-\mathrm{Hz}$ triaxial sonic anemometer velocity and air temperature measurements were carried out in the unstable ASL for a wide range of atmospheric stability conditions.

\section{Theory}

This section presents the theory underlying the dissipation and flux-variance methods in two parts. Variations on these methods are discussed in corresponding subsections in order to facilitate comparisons between predictions and measurements.

\subsection{Dissipation Method}

This method involves the development of budget equations for TKE and temperature variance. For practical applications, it is necessary to carry out simplifications to these budget equations in order to relate the flux to the mean dissipation rate at a point in the flow. These simplifications are considered in the following sections.

2.1.1. Turbulent kinetic energy (TKE) budget. By choosing a coordinate system such that the $x$ component is along the mean wind direction and assuming horizontally homogeneous and steady state conditions, the turbulent kinetic energy budget per unit mass of fluid can be expressed as

$$
-\overline{u w} \frac{\partial U}{\partial z}+\frac{g}{T} \overline{w \theta}-\frac{1}{2} \frac{\partial \overline{w e^{2}}}{\partial z}-\frac{1}{\rho} \frac{\partial \overline{p w}}{\partial z}-\varepsilon=0,
$$

where $e^{2}=u^{2}+v^{2}+w^{2}, u, v$, and $w$ are the longitudinal, lateral, and vertical velocity fluctuations along the $x, y$, and $z$ directions, respectively, $U$ is the mean horizontal wind speed, $T$ is the mean air potential temperature, $\theta$ is the fluctuation of air potential temperature, $g\left(=9.8 \mathrm{~m} \mathrm{~s}^{-2}\right)$ is the gravitational acceleration, $z$ is the height above the surface, $\rho$ is the mean density, $p$ is the pressure fluctuation, $\varepsilon$ is the mean dissipation rate of turbulent kinetic energy, and overbar denotes time averaging. Assuming the flux divergence terms (i.e., the third and fourth terms) are negligible [e.g., Deacon, 1988; Fairall and Larsen, 1986; Dyer and Hicks, 1982; Wyngaard and Cote, 1971; Busch and Panofsky, 1968], (1) simplifies to

$$
-\overline{u w} \frac{\partial U}{\partial z}+\frac{g}{T} \overline{w \theta}=\varepsilon .
$$

From MOST, the dimensionless wind shear function $\phi_{m}$ is given by

$$
\phi_{m}=\frac{k z}{u_{*}} \frac{\partial U}{\partial z}
$$

where $u_{*}$ is defined as $\overline{-u w}^{1 / 2}$ and $k(=0.4)$ is von Karman constant. The Monin-Obukhov stability length $(L)$ is defined as

$$
L=-\frac{\rho C_{p} T u_{*}^{3}}{k g H},
$$

where $H$ is equal to $\rho C_{p} \overline{w \theta}$, and $C_{p}\left(=1005 \mathrm{~J} \mathrm{Kg}^{-1} \mathrm{~K}^{-1}\right)$ is the specific heat capacity of dry air at constant pressure. Combining (2) and (3) with the definition of $L, u_{*}$ is given by

$$
u_{*}=\left[\frac{\varepsilon k z}{\phi_{m}-z / L}\right]^{1 / 3} .
$$

As suggested by Dyer [1974], $\phi_{m}$ is given as

$$
\phi_{m}=(1-16 z / L)^{-1 / 4} \quad 0<-z / L<2
$$

for unstable conditions. Hence (4) can be used to estimate $u_{*}$ if $\varepsilon$ is known. In many ASL experiments, the second-order longitudinal velocity structure function $\left(D_{u u}\right)$, given by

$$
D_{u u}(r)=\overline{[u(x+r)-u(x)]^{2}}=4 \alpha \varepsilon^{2 / 3} r^{2 / 3},
$$

is used to estimate $\varepsilon$, where $r$ is the separation distance along the $x$ direction, and $\alpha$ is the Kolmogorov constant for the one-dimensional $u$ spectrum taken as 0.55 [Kaimal and Finnigan, 1994, p. 64; Busch, 1973].

2.1.2. Temperature variance budget. Using the same assumptions of the TKE budget discussed in section 2.1.1, the temperature variance budget is given by

$$
-\overline{w \theta} \frac{\partial T}{\partial z}-\frac{1}{2} \frac{\partial \overline{w \theta^{2}}}{\partial z}-N=0,
$$

where $N$ is the mean temperature variance dissipation rate for $1 / 2 \overline{\theta^{2}}$. Assuming the flux divergence term (i.e., the second term) is negligible [e.g., Fairall and Larsen, 1986; Dyer and Hicks, 1982; Champagne et al., 1977; Wyngaard and Cote, 1971; Busch and Panofsky, 1968], (6) can be written as

$$
-\overline{w \theta} \frac{\partial T}{\partial z}=N
$$

According to MOST, the dimensionless temperature gradient $\phi_{h}$ is

$$
\phi_{h}=-\frac{k z}{T_{*}} \frac{\partial T}{\partial z}
$$

where $T_{*}\left(=H / \rho C_{p} u_{*}\right)$ is a temperature scale. Combination of (7) and (8) yields

$$
H=\rho C_{p}\left[\frac{k z u_{*} N}{\phi_{h}}\right]^{1 / 2} .
$$

Notice that (9) requires an estimate of $\phi_{h}$ and $N$ in order to estimate $H$. As suggested by Dyer [1974], $\phi_{h}$ is given by

$$
\phi_{h}=(1-16 z / L)^{-1 / 2} \quad 0<-z / L<2
$$

in unstable conditions. Also, $N$ can be determined from the second-order temperature structure function

$$
D_{\theta \theta}(r)=\overline{[\theta(x+r)-\theta(x)]^{2}}=4 \beta N \varepsilon^{-1 / 3} r^{2 / 3},
$$

where $\beta$ is the Kolmogorov constant for the one-dimensional $\theta$ spectrum taken as 0.8 [Busch, 1973].

2.1.3. Determination of $\boldsymbol{u}_{*}$ and $\boldsymbol{H}$. In the dissipation method, there are two approaches to estimate $u_{*}$ and $H$.

Method 1 ( $u$ and $\theta$ given): If both high frequency temperature and longitudinal velocity fluctuations are measured, then the following procedure can be used to estimate $u_{*}$ and $H$ : 
1. From the high-frequency velocity and temperature measurements and Taylor's frozen turbulence hypothesis, the structure functions, $D_{u и}$ and $D_{\theta \theta}$, are determined.

2. According to (5), $\varepsilon$ is determined by applying a regression analysis between the measured $\left(\overline{[u(x+r)-u(x)]^{2}}\right)$ and predicted $\left(4 \alpha \varepsilon^{2 / 3} r^{2 / 3}\right)$ structure functions. Hence, with $\varepsilon$ determined from (5), $N$ can be determined from (10) by regression analysis.

3. Using (4) and (9), $u_{*}$ and $H$ are then determined iteratively.

Method 2 ( $\boldsymbol{U}$ and $\boldsymbol{\theta}$ given): If only mean wind velocity $U$ and high-frequency temperature fluctuations are measured, $u_{*}$ and $H$ can be determined as follows.

1. From MOST, the mean wind profile can be written as [Brutsaert, 1982, pp. 66-70]

$$
U=\frac{u_{*}}{k}\left[\ln \left(\frac{z}{z_{0}}\right)-\psi_{m}\left(\frac{z}{L}\right)\right],
$$

where $z_{0}$ is the momentum roughness length and $\psi_{m}\left(\equiv \int_{0}^{z / L}\right.$ [1 $\left.\left.-\phi_{m}(x)\right] d x / x\right)$ is a stability correction function. For unstable conditions, $\psi_{m}$ can be expressed as

$$
\begin{aligned}
\psi_{m}= & 2 \ln [(1+x) / 2]+\ln \left[\left(1+x^{2}\right) / 2\right] \\
& -2 \arctan (x)+\pi / 2
\end{aligned}
$$

where $x=(1-16 z / L)^{1 / 4}$. Hence, if $z_{0}$ is known, $u_{*}$ can be computed from

$$
u_{*}=\frac{U k}{\ln \left(z / z_{0}\right)-\psi_{m}(z / L)} .
$$

2. Once $u_{*}$ is estimated from (12), $\varepsilon$ can be estimated from the definition of the dimensionless dissipation function $\phi_{\varepsilon}$, which is given by

$$
\varepsilon=\frac{u_{*}^{3}}{k z} \phi_{\varepsilon}
$$

The relation between $\phi_{\varepsilon}$ and $\phi_{m}$ can be derived by multiplying all terms in (1) by $k z / u_{*}{ }^{3}$ to obtain a dimensionless form of TKE given by $\phi_{\varepsilon}=\phi_{m}-z / L-\phi_{d}$ [Frenzen and Vogel, 1992; Schacher et al., 1981], where $\phi_{d}$ is the sum of the dimensionless divergence terms. For unstable conditions, the following formulas for $\phi_{\varepsilon}$ (see Panofsky and Dutton [1984, p. 180] and Brutsaert [1982, p. 193] for a review) have been considered in this study.

$$
\begin{array}{ll}
\phi_{\varepsilon}=1-z / L & 0<-z / L \\
\phi_{\varepsilon}=\phi_{m}-z / L & 0<-z / L<2 \\
\phi_{\varepsilon}=\left(1+0.5|z / L|^{2 / 3}\right)^{3 / 2} & 0<-z / L<2
\end{array}
$$

We should note that (14a) and (14c) are empirical formulas from measurements carried out over land surfaces, while (14b) is derived by neglecting $\phi_{d}$. In Figure 1, comparisons among these formulas are shown. Notice that the three formulas for $\phi_{\varepsilon}$ are very comparable for $-z / L<2$; however, formula (14c) significantly departs from formulas (14a) and (14b) for freeconvective conditions.

3. With $\varepsilon$ estimated from (13), the temperature structure function, that is, (10), is used to estimate $N$, and then (9) is used to estimate $H$.

Hence, if $U$ and high-frequency temperature fluctuations are

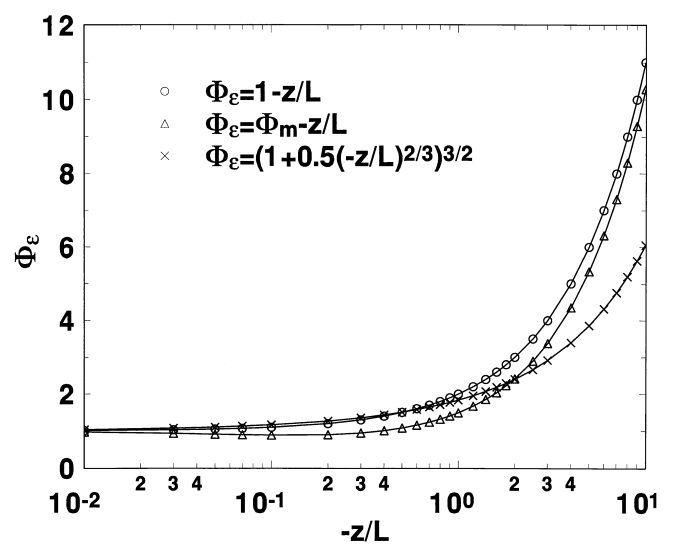

Figure 1. Comparison for the dimensionless dissipation functions $\phi_{\varepsilon}$.

measured, $u_{*}$ and $H$ can be determined by solving (12), (13), (10), and (9) iteratively.

\subsection{Flux-Variance Method}

On the basis of MOST, for the unstable ASL over a homogeneous and flat surface, the normalized standard deviation function for temperature, $\phi_{\theta}\left(\equiv \sigma_{T} / T_{*}\right)$, can be expressed as [Tillman, 1972]

$$
\phi_{\theta} \equiv \frac{\sigma_{T}}{T_{*}}=C_{1}\left(C_{2}-z / L\right)^{-1 / 3} \quad-z / L>0
$$

where $\sigma_{T}$ is the standard deviation of temperature and $C_{1}$ and $C_{2}$ are similarity constants. As $-z / L$ approaches zero, (15) converges to a constant $C_{3}\left(=\left(C_{1} / C_{2}^{1 / 3}\right)\right.$. For free convection, Wyngaard et al. [1971] showed that (15) reduces to

$$
\sigma_{T} / T_{*}=C_{1}(-z / L)^{-1 / 3}
$$

We note that the free-convection limit is not well defined [see, e.g., Kohsick, 1982; Tillman, 1972; Wyngaard et al., 1971], yet (16) appears to be accurate for a wide range of atmospheric stability conditions [e.g., Katul et al., 1995; Albertson et al., 1995, Lloyd, 1991; Padro, 1993]. Also, the value of $C_{3}$ is very uncertain [e.g., Weaver, 1990], and thus no added benefit is gained by using (15) instead of (16). Table 1 lists the values of $C_{1}, C_{2}$, and $C_{3}$ used by other investigators. On the basis of Table 1 , we recommend the use of (16) with $C_{1}=0.95$. With the definitions of $L$ and $T_{*}$, (16) leads to

$$
H=\rho C_{p}\left[\frac{\sigma_{T}}{C_{1}}\right]^{3 / 2}\left[\frac{k g z}{T}\right]^{1 / 2},
$$

which is independent of $u_{*}$, and thus $H$ can be conveniently determined from $\sigma_{T}$ only.

If $u_{*}$ is required, two approaches are proposed depending on the measured variables.

Method 1 ( $\sigma_{w}$ and $\sigma_{T}$ given): If the standard deviation of the vertical velocity $\left(\sigma_{w}\right)$ and $\sigma_{T}$ are measured, then an approach based on MOST can be implemented. Using the normalized standard deviation relation [Panofsky and Dutton, 1984, p. 161],

$$
\frac{\sigma_{w}}{u_{*}}=1.25(1-3 z / L)^{1 / 3} \quad-z / L>0
$$


Table 1. Summary of the Constants Used in $\sigma_{T} / T_{*}=C_{1}\left(C_{2}-z / L\right)^{-1 / 3}$

\begin{tabular}{|c|c|c|c|c|c|c|c|}
\hline Authors & $C_{1}$ & $\mathrm{~F}-\mathrm{C}$ & $C_{2}$ & $C_{3}$ & $-z / L$ & Site & Comment \\
\hline Tillman [1972] & 0.95 & $>0.2$ & 0.05 & 2.5 & NA & $\begin{array}{l}\text { coastal site, Mass.; sensors at } \\
16 \text { and } 40 \mathrm{~m} .\end{array}$ & $\begin{array}{l}\text { free-convection range is not rigorous; } \\
\text { Kansas and Utah data were also } \\
\text { used; } k=0.35 \text {. }\end{array}$ \\
\hline Wesely [1988] & 1.25 & $>0.31$ & NA & 1.85 & $<0.31$ & $\begin{array}{l}\text { grass and partially bare soil, } \\
\text { Germany. sensors at } 7.7 \mathrm{~m} .\end{array}$ & $\begin{array}{l}C_{1}=1.25 \text { was chosen to conform } \\
\text { with Wesely's [1983] results for } \\
\text { water vapor, } \mathrm{O}_{3} \text { and } \mathrm{CO}_{2} ; k=0.4 \text {. }\end{array}$ \\
\hline Weaver [1990] & 1.0 & $>0.1$ & NA & 2.2 & $<0.1$ & $\begin{array}{l}\text { prairie, Kansas. 7/1-7/4, } 1987 \\
z_{0}=3 \mathrm{~cm}\end{array}$ & $\begin{array}{l}\text { flux-variance relation is not universal } \\
\text { with surface and time; } k=0.4 \text {. }\end{array}$ \\
\hline Lloyd [1991] & 0.95 & NA & NA & NA & NA & $\begin{array}{l}\text { bare soil, millet, savannah, } \\
\text { bush, West Africa; sensors } \\
\text { at } 3.3-25 \mathrm{~m} .\end{array}$ & $\begin{array}{l}\text { flux-variance is universal for surfaces } \\
\text { considered. heat flux estimation is } \\
\text { influenced by the zero-plane } \\
\text { displacement; } k=0.4 \text {. }\end{array}$ \\
\hline de Bruin et al. [1993] & 0.95 & $\gg 1$ & 0.035 & 2.9 & NA & $\begin{array}{l}\text { plain, France; sensors at } \\
11.3 \mathrm{~m} .\end{array}$ & $\begin{array}{l}\text { constants are obtained from } \sigma_{T} / T_{*}= \\
\quad a[1-(b z / L)]^{-1 / 3} ; k=0.4 .\end{array}$ \\
\hline Padro [1993] & 0.95 & NA & 0.05 & 2.5 & NA & $\begin{array}{l}\text { forest }(18 \mathrm{~m}) \text {, Canada; sensors } \\
\text { at } 33.4 \mathrm{~m} .\end{array}$ & $\begin{array}{c}\sigma_{T} / T_{*} \text { varies from surface to surface. } \\
C_{3} \text { is not a constant, even in } \\
-z / L<0.001 ; k=0.4\end{array}$ \\
\hline Kustas et al. [1994] & 1.1 & NA & 0.085 & 2.5 & NA & $\begin{array}{l}\text { METFLUX site } 7 \text { (shrub- } \\
\text { dominated), Arizona; } \\
z_{0}=3 \mathrm{~cm} \text {; eddy } \\
\text { correlation system at } 2 \mathrm{~m} .\end{array}$ & $\begin{array}{l}\text { constants are adopted for the other } \\
\text { METFLUX sites; } k=0.4 \text {. }\end{array}$ \\
\hline Albertson et al. [1995] & 0.97 & NA & NA & NA & NA & $\begin{array}{l}\text { Owens Lake bed, California; } \\
z_{0}=0.13 \mathrm{~mm} \text {; triaxial } \\
\text { sonic anemometer at } 2.5 \mathrm{~m} .\end{array}$ & $\begin{array}{l}\text { measurements followed } \sigma_{T} / T_{*}= \\
C_{1}(-z / L)^{-1 / 3} \text { well below the } \\
\text { strictly defined free convection } \\
\text { region; } k=0.4 \text {. }\end{array}$ \\
\hline
\end{tabular}

Values in the $-z / L$ column represent the stability range where $\sigma_{T} / T_{*}$ converges to a constant $C_{3}\left(=\left(C_{1} / C_{2}^{1 / 3}\right)\right.$. The F-C column represents the free convection range. NA indicates that the constant was not available; $z_{0}$ is the roughness length. Hicks [1981], Panofsky and Dutton [1984], Hogstrom and Smedman-Hogstrom [1974], Monji and Businger [1972], and Wyngaard et al. [1971] report that $C_{1}$ is around unity [see Wesely, 1988]. METFLUX, meteorological energy flux.

combined with the definition of $L$ results in an explicit expression for $u_{*}$ given by

$$
u_{*}=\left[\left(\frac{\sigma_{w}}{1.25}\right)^{3}-\frac{3 k g H z}{\rho C_{p} T}\right]^{1 / 3} .
$$

With $H$ estimated from (17), $u_{*}$ can be estimated from (19).

Method 2 ( $U$ and $\sigma_{T}$ given): If the mean wind speed, temperature standard deviation, and momentum roughness length $z_{0}$ are given, then $u_{*}$ can be estimated by solving (12) and (17) iteratively.

\section{Experiment}

The experiment was carried out over an Alta Fescue grasscovered forest clearing at the Blackwood division of the Duke Forest in Durham, North Carolina. The site is approximately $480 \mathrm{~m}$ by $305 \mathrm{~m}$ surrounded by $10-\mathrm{m}$ Loblolly pine stand (the site elevation is $163 \mathrm{~m}$ ). The mast was located at $50 \mathrm{~m}$ from the north edge and $100 \mathrm{~m}$ from the west edge of the forest clearing. The three wind velocity components and air temperature were measured at $z=1.54 \mathrm{~m}$ above the ground surface using a Gill triaxial sonic anemometer. The air temperature was determined from the sound speed $C_{s}$ using $\left(C_{s}\right)^{2}=\gamma R_{d} T$, where $R_{d}\left(=287.04 \mathrm{~J} \mathrm{~kg}^{-1} \mathrm{~K}^{-1}\right)$ is the gas constant of dry air at constant pressure, $\gamma(=1.4)$ is the ratio of the molar specific heat capacities of air at constant pressure to that at constant volume. Using Taylor's hypothesis $(r=U t)$, the second-order structure function for a flow variable $(s)$ is determined from

$$
D_{s s}(U t)=\overline{[s(x+U t)-s(x)]^{2}},
$$

where $s=u$ or $T$.
The measured mean grass height was $23 \mathrm{~cm}$. The momentum roughness length $z_{0}$ was calculated from (11a) by using the measured $u_{*}$ and $U$ for near-neutral atmospheric conditions and neglecting the zero-plane displacement height. The resulting mean $z_{0}$ for this grass site was $6.5 \mathrm{~cm}$. Two data sets collected in July and August 1994 were used for comparing the dissipation and flux-variance predictions with the eddy correlation measurements.

The first data set was collected on July 26 and 27, 1994. The weather was sunny during these 2 days with some clouds noted in the late afternoon. This portion of the experiment includes 26 runs, each having a sampling frequency and duration of 21 $\mathrm{Hz}$ and $26 \mathrm{~min}$, respectively, resulting in 32,768 data points per measured variable. The 26 individual runs were inspected for (1) trends and unsteadiness in the mean meteorological conditions, (2) low mean wind speeds $\left(<0.4 \mathrm{~m} \mathrm{~s}^{-1}\right)$, and (3) large standard deviation in the wind directions. This inspection was necessary to insure that the variances and structure function measurements were not contaminated by variability in the mean meteorological conditions or large fluctuations in wind direction. Also, the applicability of Taylor's frozen turbulence hypothesis requires low turbulent intensity that is typically not satisfied at very low wind speeds. After this preliminary inspection, only 19 runs satisfied the above criteria.

The second data set was collected on August 10, 1994. Clearsky conditions were noted for the majority of daylight hours. The sampling frequency and duration for each run were $56 \mathrm{~Hz}$ and $19.5 \mathrm{~min}$ resulting in 65,536 data points per measured variable. This experiment resulted in 20 runs of which 17 satisfied the above criteria. Hence the two combined experiments resulted in 36 runs. 


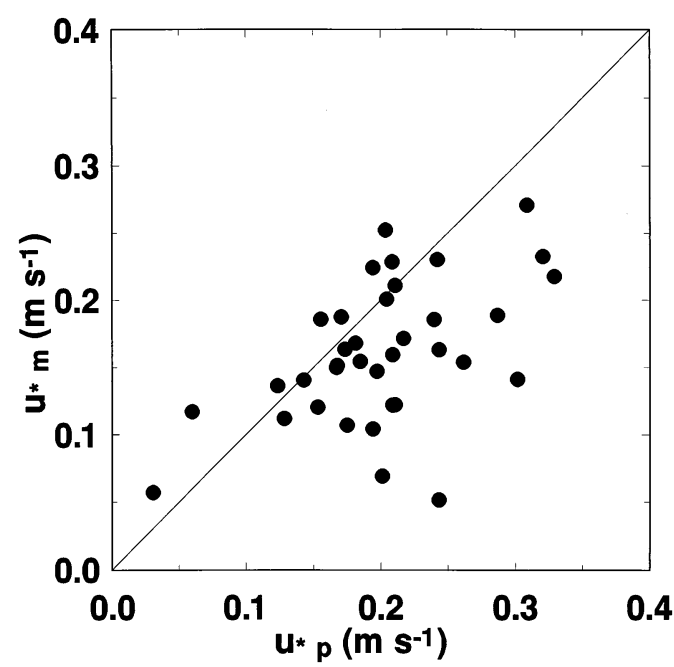

Figure 2a. Comparison between dissipation method $1(u$ and $\theta$ given) predicted $\left(u_{* p}\right)$ and eddy correlation measured $\left(u_{* m}\right)$ friction velocity. The 1:1 line is also shown.

\section{Results and Discussion}

\subsection{Friction Velocity $\boldsymbol{u}_{*}$}

Figure 2a shows the comparison between dissipation method 1 ( $u$ and $\theta$ given) predicted $\left(u_{* p}\right)$ and eddy correlation measured $\left(u_{* m}\right)$ friction velocity. Figure $2 \mathrm{~b}$ is the same as Figure $2 \mathrm{a}$, but using dissipation method 2 ( $U$ and $\theta$ given). Figures $3 \mathrm{a}$ and $3 \mathrm{~b}$ also show the friction velocity comparisons, but using flux-variance method 1 ( $\sigma_{w}$ and $\sigma_{T}$ given) and method 2 ( $U$ and $\sigma_{T}$ given), respectively. Fair agreement between predictions and measurements is noted in Figures $2 \mathrm{a}$ to $3 \mathrm{~b}$. This, in part, is due to the following:

1. The surface is nonuniform and MOST assumptions are violated. Notice that in dissipation methods 1 and 2 and fluxvariance method $2, \phi_{m}$ and $\psi_{m}$ play key roles in estimating $u_{*}$ (recall dissipation method 1 uses (4), and dissipation method 2 and flux-variance method 2 use (12) to estimate $u_{*}$ ). For flux-variance method $1, \sigma_{w} / u_{*}$ plays a key role in estimating

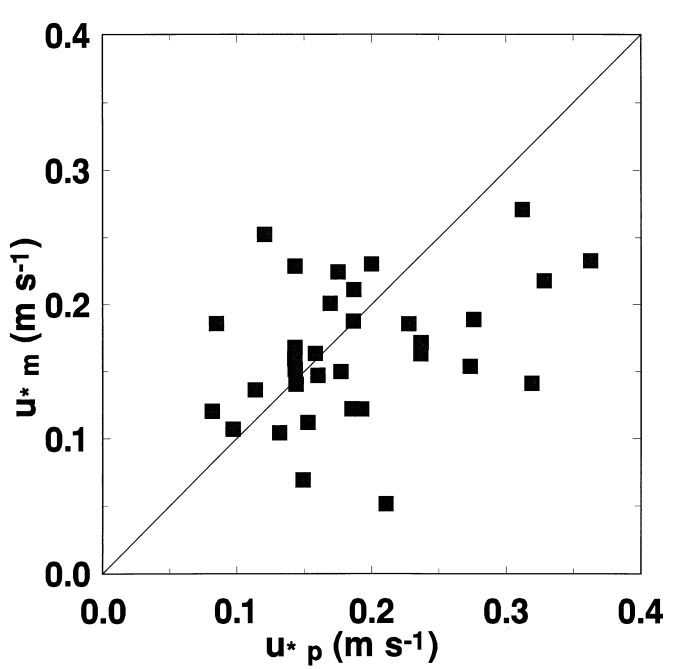

Figure 3a. Comparison between flux-variance method $1\left(\sigma_{w}\right.$ and $\sigma_{T}$ given) predicted $\left(u_{* p}\right)$ and eddy correlation measured $\left(u_{* m}\right)$ friction velocity. The $1: 1$ line is also shown.

$u_{*}$ (recall flux-variance method 1 uses (19) to estimate $u_{*}$ ). Hence it is important to examine the validity of $\phi_{m}, \psi_{m}$, and $\sigma_{w} / u_{*}$ derived above uniform surfaces for this specific site. While $\phi_{m}$ was not directly measured in this experiment, $\psi_{m}$ can be taken as a good surrogate for $\phi_{m}$ since $\psi_{m} \equiv$ $\int_{0}^{z / L}\left[1-\phi_{m}(x)\right] d x / x$. Figure 4a presents the comparison between predicted (solid line, calculated from (11b)) and measured (circles, calculated from (11a) with measured $U$ and $u_{*}$ ) $\psi_{m}$ as a function of $-z / L$. Figure $4 \mathrm{~b}$ shows the comparison between the measured and predicted $\sigma_{w} / u_{*}$. The scatter in Figures $4 \mathrm{a}$ and $4 \mathrm{~b}$ shows that $\phi_{m}, \psi_{m}$, and $\sigma_{w} / u_{*}$ deviate from MOST predictions which partly explains the departure between measured and predicted $u_{*}$. Also, notice in Figure $2 \mathrm{~b}$ that the estimated $u_{*}$ is not sensitive to the different formulas for $\phi_{\varepsilon}$ as evidenced earlier in Figure 1.

2. The uncertainty in eddy correlation measurement. Notice that measured $u_{*}$ are small $\left(<0.3 \mathrm{~m} \mathrm{~s}^{-1}\right)$ and are suscep-

tible to large measurement errors.

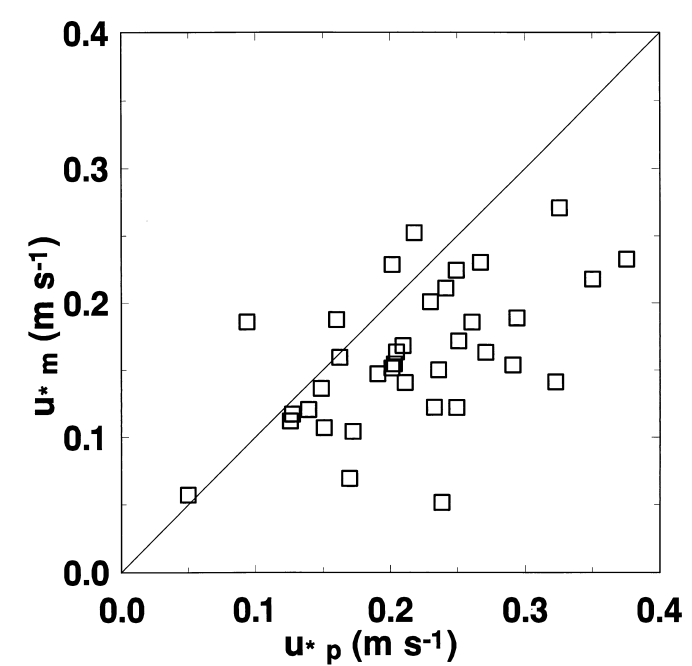

Figure 3b. Comparison between flux-variance method 2 ( $U$ and $\sigma_{T}$ given) predicted $\left(u_{* p}\right)$ and eddy correlation measured $\left(u_{* m}\right)$ friction velocity. The 1:1 line is also shown.
Figure 2b. Comparison between dissipation method 2 ( $U$ and $\theta$ given) predicted $\left(u_{* p}\right)$ and eddy correlation measured $\left(u_{* m}\right)$ friction velocity. The 1:1 line is also shown.

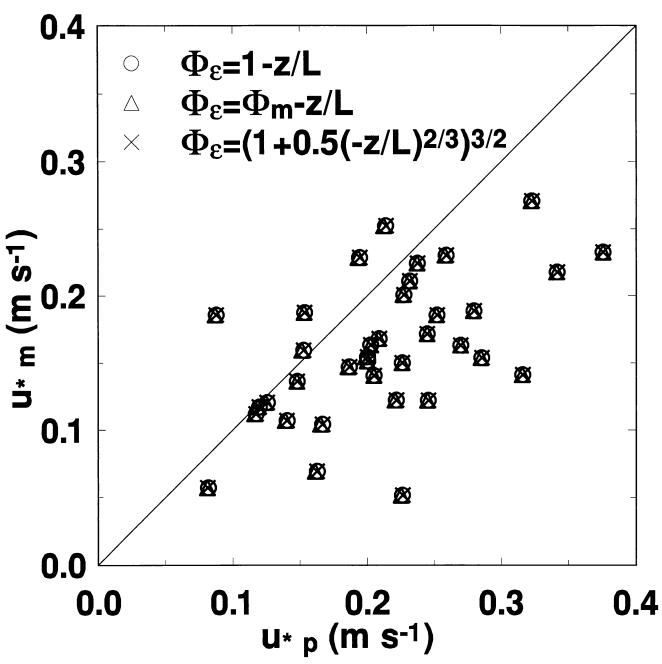




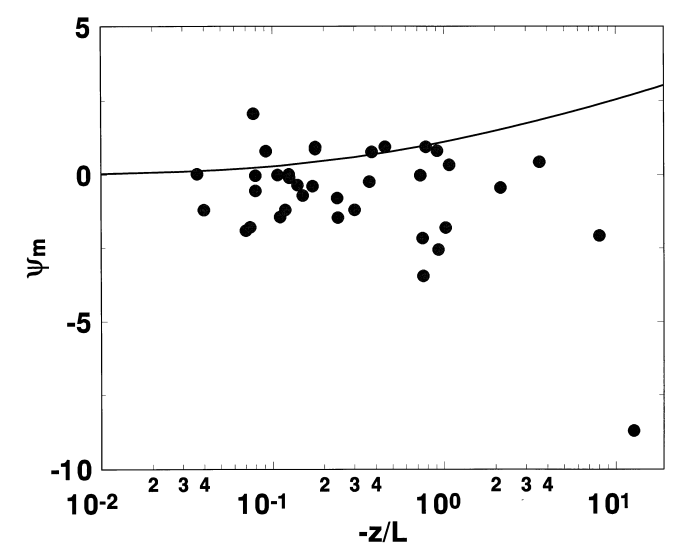

Figure 4a. Comparison between predicted (solid line, calculated from (11b)) and measured (circles, calculated from (11a) with measured $U$ and $\left.u_{*}\right) \psi_{m}$ as a function of $-z / L$.

\subsection{Sensible Heat Flux $\boldsymbol{H}$}

Figure 5a presents the comparison between dissipation method 1 predicted $\left(H_{p}\right)$ and eddy correlation measured $\left(H_{m}\right)$ sensible heat flux. In this comparison, (4) in conjunction with (9) were used to determine $H_{p}$. Figure $5 \mathrm{~b}$ is the same as Figure 5a but using dissipation method 2, where (9) in conjunction with (12) were used to estimate $H_{p}$. Notice that the three different formulas of $\phi_{\varepsilon}$ result in equivalent predictions. It is evident from Figures $5 \mathrm{a}$ and $5 \mathrm{~b}$ that the dissipation method predictions are in good agreement with the eddy correlation measurements. The good agreement indicates that $\phi_{h}$ is less sensitive to heat nonuniformity and the formula given by Dyer [1974] is valid for this experiment.

Figure 6a is the same as Figure 5a but for the flux-variance method. Notice in Figure $6 a$ that the flux-variance method systematically overestimates the sensible heat flux. These results suggest that $T_{*}$ is systematically overestimated. The consequences of this overestimation is considered next in Figure $6 b$.

In Figure 6b, the measured and predicted $(0.95$ $\left.(-z / L)^{-1 / 3}\right) \sigma_{T} / T_{*}$ are shown as a function of $-z / L$. Using Figure $6 \mathrm{~b}$, we note the following:

1. The measured $\sigma_{T} / T_{*}$ is larger than the predicted value for a wide range of $z / L$. This can be attributed to the variability

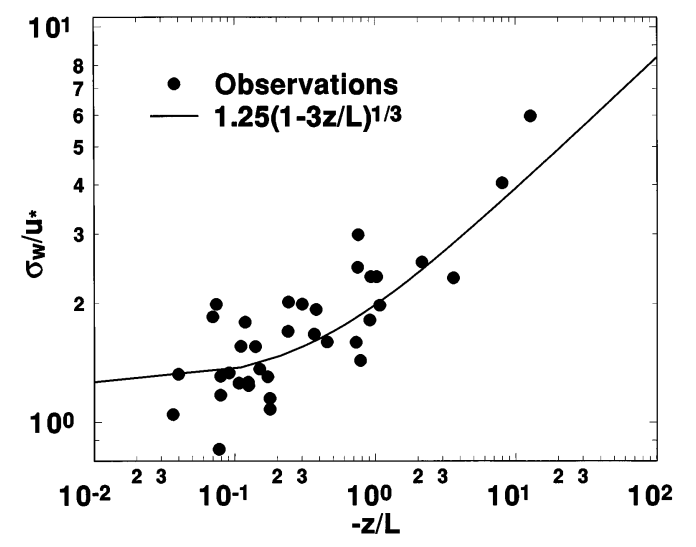

Figure 4b. Comparison between eddy correlation measured and predicted $\sigma_{w} / u_{*}$ as a function of $-z / L$. The predicted $\sigma_{w} / u_{*}$ is calculated by (18).

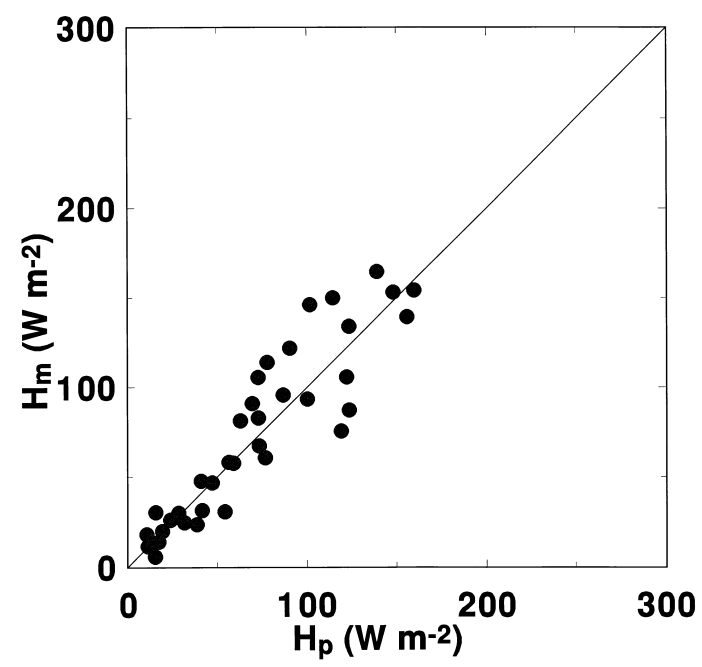

Figure 5a. Comparison between dissipation method 1 ( $u$ and $\theta$ given) predicted $\left(H_{p}\right)$ and eddy correlation measured $\left(H_{m}\right)$ sensible heat flux. The 1:1 line is also shown.

in sources and sinks of heat at the ground level that produce additional air temperature variance not explained by the surface fluxes $H$ and $u_{*}$ (and thus $T_{*}$ ). That is, $\sigma_{T}$ is the result of mean surface fluxes $\left(T_{*}\right)$ as well as variability in heat sources and sinks at the ground level. These sources and sinks are absent in homogeneous and flat terrain that is uniformly heated. The additional variance due to nonuniformity in ground heating results in $\sigma_{T} / T_{*}>0.95(-z / L)^{-1 / 3}$. Hence $H$ estimated from the measured $\sigma_{T}$ will be larger than the eddy correlation measurements because the $\sigma_{T}$ is not simply due to the mean surface flux (as assumed by the flux-variance method) but also due to nonuniformity in ground heating.

2. The dependence of $\sigma_{T} / T_{*}$ on $(-z / L)^{-1 / 3}$ is also evident in Figure $6 b$. This suggests that the free-convective $-1 / 3$ power law is not sensitive to the nonuniformity in ground heating. Hence, as suggested by Weaver [1990], a modification to the 0.95 coefficient in (16) is suitable, and the flux-variance method may still result in accurate estimation of $H$ if $C_{1}$ is

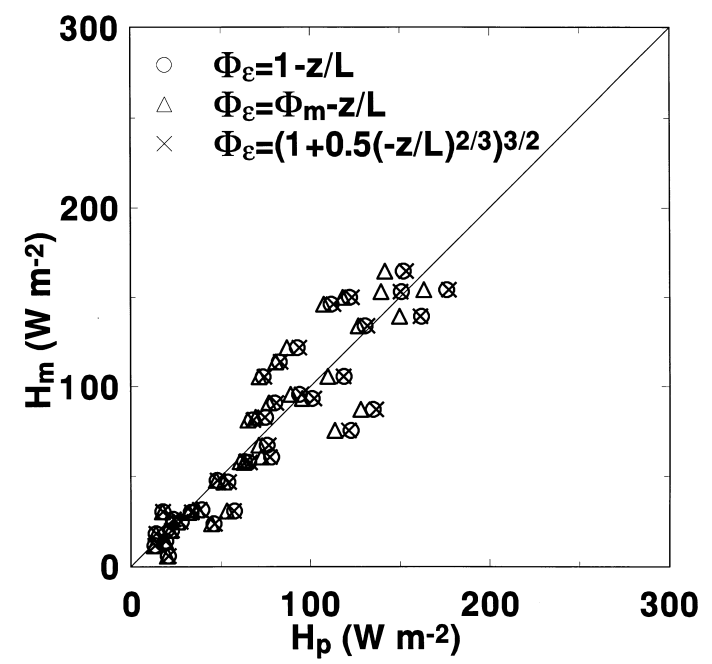

Figure 5b. Comparison between dissipation method 2 ( $U$ and $\theta$ given) predicted $\left(H_{p}\right)$ and eddy correlation measured $\left(H_{m}\right)$ sensible heat flux. The 1:1 line is also shown. 


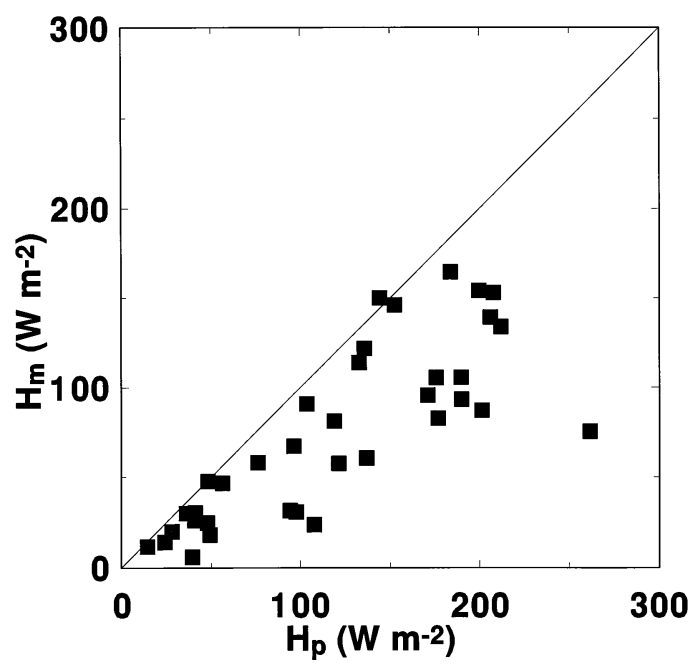

Figure 6a. Comparison between flux-variance method $\left(C_{1}=0.95\right)$ predicted $\left(H_{p}\right)$ and eddy correlation measured $\left(H_{m}\right)$ sensible heat flux. The 1:1 line is also shown.

known a priori. In fact, for nonuniform terrain, $C_{1}$ will be larger than 0.95 , depending on the level of nonuniformity in the ground heating. However, in this context, Lloyd et al. [1991] found that the universal function for $\sigma_{T} / T_{*}$ is independent of the terrain type; but no assessment on the level of heat nonuniformity was reported in their study.

In order to minimize the departure between measured and predicted $H, C_{1}$ should be adjusted. For adjusting $C_{1}, \phi_{\theta}$ was modified to fit the measurements. From regression analysis, $\phi_{\theta}=1.36(-z / L)^{-1 / 3}$, that is, $C_{1}=1.36$ rather than 0.95 . Figure $6 \mathrm{c}$ presents the comparison between the flux-variance method $\left(C_{1}=1.36\right)$ predicted $\left(H_{p}\right)$ and eddy correlation measured $\left(H_{m}\right)$ sensible heat flux. Good agreement between predicted and measured $H$ indicates that 1.36 is a better coefficient than 0.95 for this specific nonuniformly ground heating condition.

According to Dyer and Hicks [1982], Weaver [1990], and the discussion above, it is evident that the dissipation and fluxvariance methods can be improved upon if the universal functions of $\phi_{m}, \phi_{h}$, and $\phi_{\theta}$ are well defined for a specific site. Since different investigators proposed different formulations

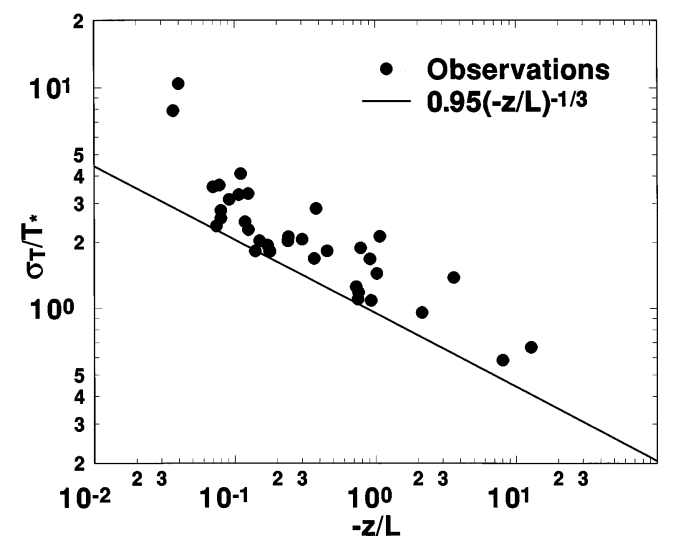

Figure 6b. Comparison between eddy correlation measured and predicted $\sigma_{T} / T_{*}$ as a function of $-z / L$. The predicted $\sigma_{T} / T_{*}$ was calculated by (16) with $C_{1}=0.95$.

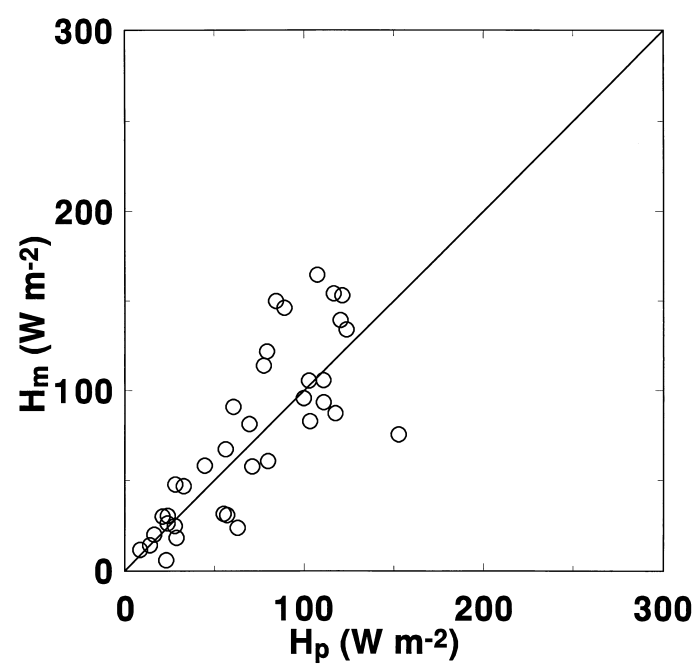

Figure 6c. Comparison between flux-variance method $\left(C_{1}=1.36\right)$ predicted $\left(H_{p}\right)$ and eddy correlation measured $\left(H_{m}\right)$ sensible heat flux. The 1:1 line is also shown.

for these universal functions, we compared all these proposed functions in Table 2 and Figures 7a and 7b. From Figures 7a and $7 \mathrm{~b}$, it is obvious that those universal functions are not in good agreement especially for momentum. Whether these differences are due to some level of nonuniformity in the momentum and heat sources and sinks at the ground is yet to be determined.

\subsection{Proposed Method for Estimating $H$}

From (7), $\overline{w \theta}$, which defines the product of a characteristic velocity and a temperature scale, is identical to $N /(\partial T / \partial z)$. On the basis of this equality and dimensional analysis [see Stull, 1988, pp. 347-348], the characteristic length, time, and temperature scales are $z, \sigma_{T}^{2} / N$, and $\sigma_{T}$, respectively. Hence $H=$ $f\left(N, z, \sigma_{T}\right)$ and can be expressed as

$$
H=\rho C_{p} \frac{G_{K} N z}{\sigma_{T}},
$$

where $G_{K}$ is a dimensionless constant.

Now, to determine the constant $\left(G_{K}\right)$ for a uniform terrain where MOST is valid, (7), (8), and (15) are combined to obtain

$$
H=\rho C_{p} \frac{N z}{\sigma_{T}}\left[\frac{\phi_{\theta}}{\phi_{h}} k\right] .
$$

By comparing (19) and (20), $G_{K}$ is given by

$$
G_{K}=\left[\frac{\phi_{\theta}}{\phi_{h}} k\right] \text {. }
$$

From (21), it is evident that $G_{K}$ can be estimated using published data sets from other ASL experiments. We focus on the extensive data set presented by Kader and Yaglom [1990] for the unstable ASL. Kader and Yaglom [1990] divided the unstable ASL into three sublayers and suggested the following formulas for $\phi_{h}$ and $\phi_{\theta}$ (these formulas are adjusted by us for a von Karman's constant $k=0.4$ in the definition of $L$ ).

$$
\phi_{h}= \begin{cases}0.96 & 0<-z / L<0.04 \\ 0.324(-z / L)^{-1 / 3} & 0.12<-z / L<1.2 \\ 0.265(-z / L)^{-1 / 3} & -z / L>2\end{cases}
$$


Table 2. Formulas for $\phi_{m}$ and $\phi_{h}$

\begin{tabular}{|c|c|c|c|c|}
\hline Authors & $\phi_{m}$ & $\phi_{h}$ & $-z / L$ & Comments \\
\hline Swinbank [1968] & $0.61(-z / L)^{-0.2}$ & $0.23(-z / L)^{-0.44}$ & $0.1-2$ & $\begin{array}{c}k=0.4, u_{*} \text { observed from drag } \\
\text { coefficient }\end{array}$ \\
\hline Zilitinkevich and Chalikov & $1+1.38 z / L$ & $0.95+1.31 z / L$ & $0-0.16$ & $k=0.4$ \\
\hline [1968] & $0.42(-z / L)^{-1 / 3}$ & $0.40(-z / L)^{-1 / 3}$ & $>0.16$ & formulas modified from $k=0.43$ \\
\hline Dyer and Hicks [1970] & $(1-16 z / L)^{-1 / 4}$ & $(1-16 z / L)^{-1 / 2}$ & $0-1$ & $k=0.41$ \\
\hline Businger et al. [1971] & $(1-15 z / L)^{-1 / 4}$ & $0.74(1-9 z / L)^{-1 / 2}$ & $0-2$ & $k=0.35$ \\
\hline Dyer and Bradley [1982] & $(1-28 z / L)^{-1 / 4}$ & $(1-14 z / L)^{-1 / 2}$ & $0.004-4$ & $k=0.4$ \\
\hline Foken and Skeib [1983] & $(-z / 0.06 L)^{-1 / 4}$ & $(-z / 0.06 L)^{-1 / 2}$ & $\begin{array}{l}0-0.06 \\
>0.06\end{array}$ & $k=0.4$ \\
\hline Kader and Yaglom [1990] & $\begin{array}{l}1.04 \\
0.50(-z / L)^{-1 / 3} \\
0.38(-z / L)^{1 / 3}\end{array}$ & $\begin{array}{l}0.96 \\
0.324(-z / L)^{-1 / 3} \\
0.265(-z / L)^{-1 / 3}\end{array}$ & $\begin{array}{l}0-0.04 \\
0.12-1.2 \\
>2\end{array}$ & $k=0.4$ \\
\hline Brutsaert [1992]* & $\left(a+b y^{\pi}\right) /\left(a+y^{\pi}\right)+c y^{1 / 3}$ & $\left(a+b y^{\pi}\right) /\left(a+y^{\pi}\right)$ & $0.01-20$ & $\begin{array}{l}k=0.4 \\
\phi_{m}(0)=\phi_{h}(0)=1\end{array}$ \\
\hline
\end{tabular}

This table combines Dyer [1974, Table 1], Hogstrom [1988, Table VI], and update from recent experiments. The $-z / L$ column indicates the suitable range for applying these formulas.

${ }^{*}$ For $\phi_{m}, y=-z / L ; a=0.37 ; b=-0.24 ; n=0.72 ; c=0.50$. For $\phi_{h}, y=-z / L ; a=0.33 ; b=0.057 ; n=0.78$.

$$
\phi_{\theta}= \begin{cases}2.9 & 0<-z / L<0.04 \\ 1.032(-z / L)^{-1 / 3} & 0.12<-z / L<1.2 \\ 1.105(-z / L)^{-1 / 3} & -z / L>2\end{cases}
$$

On the basis of these formulas, we calculated

$$
G_{K}= \begin{cases}1.21 & 0<-z / L<0.04 \\ 1.27 & 0.12<-z / L<1.2 \\ 1.67 & -z / L>2\end{cases}
$$

Hence we expect $G_{K}$ to be between 1.21 and 1.67 for a uniform terrain; however, for near-neutral conditions, values for $\phi_{\theta}$ are widely scattered [e.g., Weaver, 1990]; thus the 1.21 lower limit for $G_{K}$ is uncertain. Also, it is important to note that Kader and Yaglom [1990] did not determine $\phi_{h}$ and $\phi_{\theta}$ for the same terrain type but obtained best fit regressions from a wide range of ASL experiments, and this may cause some variation in $G_{K}$ from 1.21 to 1.67 .

To determine $G_{K}$ for our nonuniform site, a regression between $H$ and $\rho C_{p} N z / \sigma_{T}$ resulted in a $G_{K}=1.66$ for $0.03<-z / L<10\left(R^{2}=0.84\right)$ which is in agreement with Kader and Yaglom [1990] data. Figure 8 shows the good agreement between eddy correlation measured and predicted $H$ using (19) with $G_{K}=1.66$.

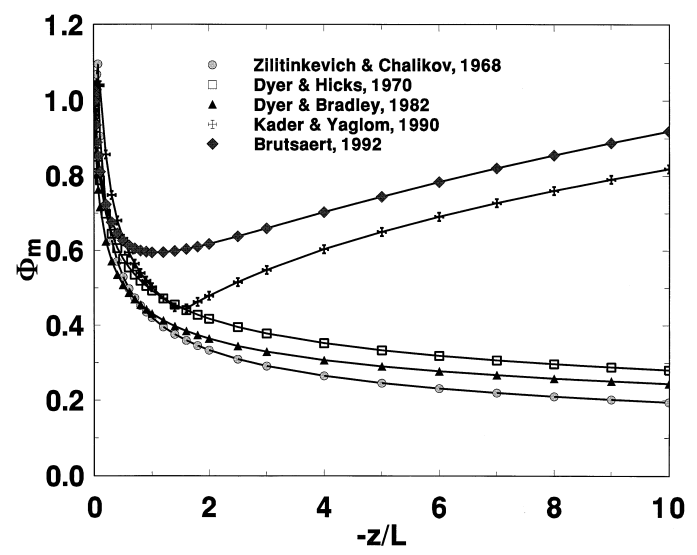

Figure 7a. Comparison among different formulas for $\phi_{m}$, see Table 2 also.
To further examine $G_{K}$, we estimate $\phi_{h}$ from (21). With $\phi_{\theta}=$ $1.36(-z / L)^{-1 / 3}, G_{K}$ in $(21)$ suggests $\phi_{h}=0.33(-z / L)^{-1 / 3}$ rather than Dyer's [1974] $\phi_{h}=(1-16 z / L)^{-1 / 2}$; however, as indicated in Figures $5 \mathrm{a}$ and $5 \mathrm{~b}, \phi_{h}=(1-16 z / L)^{-1 / 2}$ is valid for this experiment. Figure 9 compares these two $\phi_{h}$ functions with the measurements (the observed $\phi_{h}$ were calculated from $\phi_{h}=N k z \rho C_{p} / H T_{*}$ with eddy correlation measured $H$ and $T_{*}$ and $N$ determined from (10)). It is evident that in the range of $0.05<-z / L<1.0$, the differences between these two functions are small, and both relations fit the data well (theoretically, $\phi_{h}$ should scale with $(-z / L)^{-1 / 3}$ under freeconvective conditions).

\section{Conclusions}

This study compared the performance of the dissipation and flux-variance methods for estimating friction velocity and sensible heat flux above a grass site. A proposed dissipation method for estimating $H$, derived from dimensional analysis, was also tested. Our results suggest the following:

1. The estimated sensible heat flux from the dissipation method is in good agreement with the measured sensible heat flux. Also, the flux-variance method reproduced the eddy correlation sensible heat flux well following an adjustment to $C_{1}$.

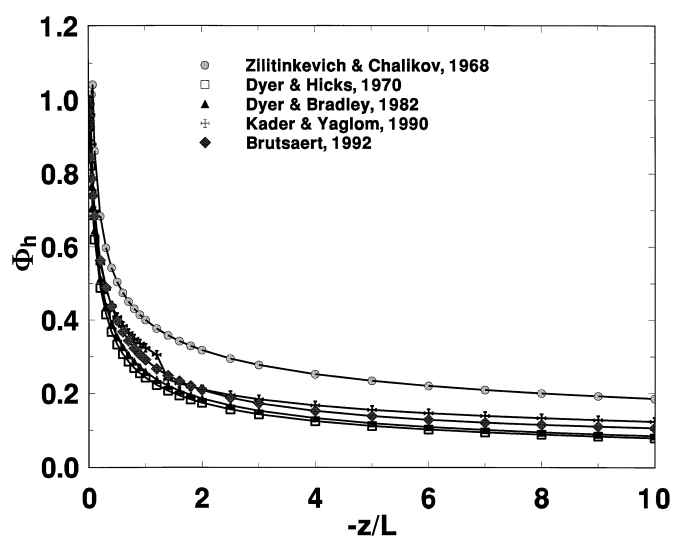

Figure 7b. Comparison among different formulas for $\phi_{h}$, see Table 2 also. 


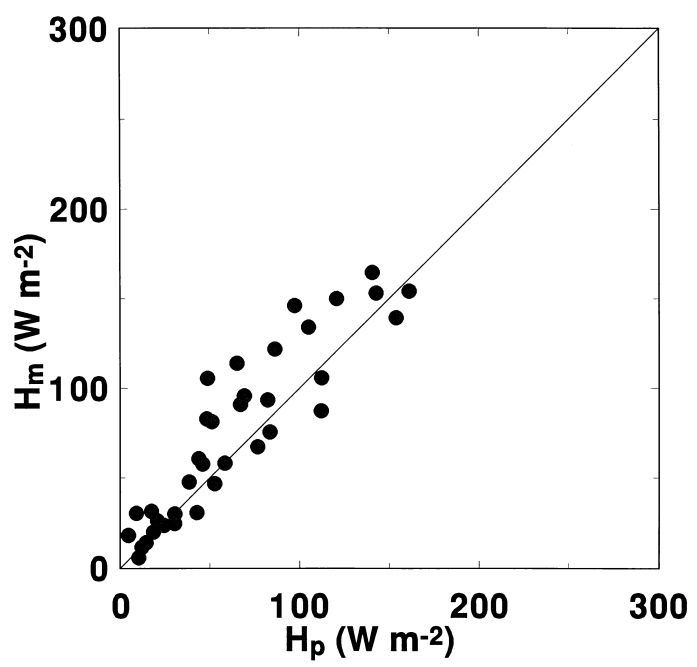

Figure 8. Comparison of sensible heat flux between eddy correlation measurements $\left(H_{m}\right)$ and predictions $\left(H_{p}\right)$ using (20) with $G_{K}=1.66$. The $1: 1$ line is also shown.

This result further confirms Weaver's [1990] conclusion that flux-variance relation has to be determined before applying flux-variance method to estimate heat flux.

2. Both dissipation and flux-variance methods did not reproduce the eddy correlation friction velocity as well as the sensible heat flux. This, in part, can be attributed to the sensitivity of the methods to $\phi_{m}, \psi_{m}$, and $\sigma_{w} / u_{*}$ which are influenced by site nonuniformity. Also, the eddy correlation $u_{*}$ are small $\left(<0.3 \mathrm{~m} \mathrm{~s}^{-1}\right)$ and are susceptible to large measurement errors.

3. On the basis of temperature variance budget equation and dimensional analysis, the sensible heat flux can be expressed as $H=\rho C_{p}\left(G_{K} N z\right) / \sigma_{T}$, where $G_{K}$ is a similarity constant. From published data above uniform terrain and the current data set, $G_{K}$ was shown to be around 1.6. Good agreement between measured and predicted $H$ was also demonstrated for a $G_{K}=1.66$. While the effect of ground heating nonuniformity produces departures between MOST predicted and the site measured $\phi_{\theta}$ as in the flux-variance method (e.g., the value of $C_{1}$ ranged from 0.95 for uniform terrain to 1.36 for our site), this nonuniformity is less likely to influence $G_{K}$ as

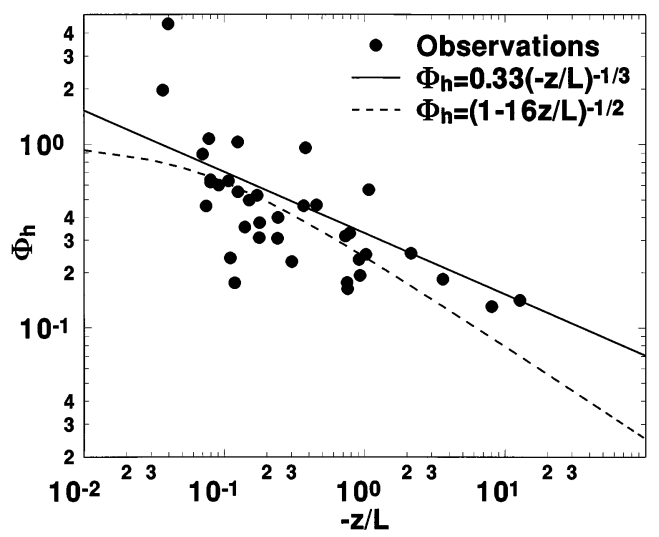

Figure 9. Comparison between measured and predicted $\phi_{h}$ as a function of $-z / L$. indicated by this experiment. However, further experiments should be performed to confirm this point.

Acknowledgments. The authors would like to thank Judd Edeburn for his help at the Duke Forest site. This project was supported, in part, by the Environmental Protection Agency (EPA) under the cooperative agreement 91-0074-94 (CR817766) and the science division of the Jet Propulsion Laboratory.

\section{References}

Albertson, J. D., M. B. Parlange, G. G. Katul, C. R. Chu, H. Stricker, and S. Tyler, Sensible heat flux from arid regions using a simple flux-variance method, Water Resour. Res., 31, 969-973, 1995.

Brutsaert, W., Evaporation into the Atmosphere: Theory, History, and Applications, 299 pp., D. Reidel, Norwell, Mass., 1982.

Brutsaert, W., Stability correction functions for the mean wind speed and temperature in the unstable surface layer, Geophys. Res. Lett., 19, 469-472, 1992.

Busch, N. E., The surface boundary layer, I, Boundary Layer Meteorol., 4, 213-240, 1973.

Busch, N. E., and H. A. Panofsky, Recent spectra of atmospheric turbulence, Q. J. R. Meteorol. Soc., 94, 132-140, 1968.

Businger, J. A., J. C. Wyngaard, Y. Izumi, and E. F. Bradley, Fluxprofile relationships in the atmospheric surface layer, J. Atmos. Sci., 28, 181-189, 1971.

Champagne, F. H., C. A. Friehe, and J. C. Larue, Flux measurements, flux estimation techniques, and Fine-Scale turbulence measurements in the unstable surface layer over land, J. Atmos. Sci., 34, 515-530, 1977.

Deacon, E. L., The measurement of turbulent transfer in the lower atmosphere, $A d v$. Geophys., 6, 211-228, 1959.

Deacon, E. L., The streamwise Kolmogroroff constant, Boundary Layer Meteorol., 42, 9-17, 1988.

de Bruin, H. A. R., Analytic solutions of the equations governing the temperature fluctuation method, Boundary Layer Meteorol., 68, 427432, 1994.

de Bruin, H. A. R., N. I. Bink, and L. J. M. Kroon, Fluxes in the surface layer under advection conditions, ed. Workshop on Land Surface Evaporation, Measurement and Parameterization, edited by T. J. Schmugge and J. C. Andre, pp. 157-169, Springer-Verlag, New York, 1991.

de Bruin, H. A. R., W. Kohsiek, and B. J. J. M. Van Den Hurk, A verification of some methods to determine the fluxes of momentum, sensible heat, and water vapor using standard deviation and structure parameter of scalar meteorological quantities, Boundary Layer Meteorol., 63, 231-257, 1993.

Dyer, A. J., A review of flux-profile relationships, Boundary Layer Meteorol., 7, 363-372, 1974.

Dyer, A. J., and E. F. Bradley, An alternative analysis of flux-gradient relationships at the 1976 ITCE, Boundary Layer Meteorol., 22, 3-19, 1982.

Dyer, A. J., and B. B. Hicks, Flux-gradient relationships in the constant flux layer, Q. J. R. Meteorol. Soc., 96, 715-721, 1970.

Dyer, A. J., and B. B. Hicks, Kolmogoroff constants at the 1976 ITCE, Boundary Layer Meteorol., 22, 137-150, 1982.

Fairall, C. W., and S. E. Larsen, Inertial-dissipation methods and turbulent fluxes at the air-ocean interface, Boundary Layer Meteorol., 34, 287-301, 1986.

Foken, T., and G. Skeib, Profile measurements in the atmospheric near-surface layer and the use of suitable universal functions for the determination of the turbulent energy exchange, Boundary Layer Meteorol., 25, 55-62, 1983.

Frenzen, P., and C. A. Vogel, The turbulent kinetic energy budget in the atmospheric surface layer: A review and an experimental reexamination in the field, Boundary Layer Meteorol., 60, 49-76, 1992.

Hogstrom, U., Non-dimensional wind and temperature profiles in the atmospheric surface layer: A re-evaluation, Boundary Layer Meteorol., 42, 55-78, 1988.

Hogstrom, U., and A. Smedman-Hogstrom, Turbulence mechanisms at an agricultural site, Boundary Layer Meteorol., 7, 373-389, 1974.

Kader, B. A., Determination of turbulent momentum and heat fluxes by spectral methods. Boundary Layer Meteorol., 61, 323-347, 1992.

Kader, B. A., and A. M. Yaglom, Mean fields and fluctuation moments 
in unstable stratified turbulent boundary layers, J. Fluid Mech., 212, 637-662, 1990.

Kaimal, J. C., and J. J. Finnigan, Atmospheric Boundary Layer Flows, 289 pp., Oxford Univ. Press, New York, 1994.

Katul, G. G., M. Goltz, C. Hsieh, Y. Cheng, F. Mowry, and J. Sigmon, Estimation of surface heat and momentum fluxes using the fluxvariance method above uniform and non-uniform terrain, Boundary Layer Meteorol., 74, 237-260, 1995.

Kohsick, W., Measuring $C_{T}^{2}, C_{Q}^{2}$ and $C_{T Q}$ in the unstable surface layer and the relations to the vertical fluxes of heat and moisture, Boundary Layer Meteorol., 24, 89-107, 1982.

Kustas, W. P., J. H. Blanford, D. I. Stannard, C. S. T. Daughtry, W. D. Nichols, and M. A. Weltz, Local energy flux estimates for unstable conditions using variance data in semiarid rangelands, Water Resour. Research., 30, 1351-1361, 1994.

Lloyd, C. R., A. D. Culf, A. J. Dolman, and J. H. C. Gash, Estimates of sensible heat flux from observations of temperature fluctuations, Boundary Layer Meteorol., 57, 311-322, 1991.

Monji, N., and J. A. Businger, Stability dependent of temperature, humidity and vertical wind variances in the atmospheric surface layer, J. Meteorol. Soc. Jpn., 50, 122-130, 1972.

Monin, A. S., and A. M. Obukhov, Basic laws of turbulent mixing in the ground layer of the atmosphere, Tr. Geofiz. Inst. Akad. Nauk. SSSR, 151, 163-187, 1954.

Padro, J., An investigation of flux-variance methods and universal functions applied to three land-use types in unstable conditions, Boundary Layer Meteorol., 66, 413-425, 1993.

Padro, J., G. den Hartog, H. H. Neumann, and D. Woolridge, Using measured variances to compute surface fluxes and dry deposition velocities: A comparison with measurements from three surface types, Atmos. Ocean, 30, 363-382, 1992.

Panofsky, H., and J. Dutton, Atmospheric Turbulence: Models and Methods for Engineering Applications, 397 pp., John Wiley, New York, 1984.

Schacher, G. E., K. L. Davidson, T. Houlihan, and C. W. Fairall, Measurements of the rate of dissipation of turbulent kinetic energy, $\varepsilon$, over the ocean, Boundary Layer Meteorol., 20, 321-330, 1981.
Stull, R. B., An Introduction to Boundary Layer Meteorology, 666 pp., Kluwer Acad., Norwell, Mass., 1988.

Swinbank, W. G., A comparison between predictions of dimensional analysis for the constant flux layer and observations in unstable conditions, Q. J. R. Meteorol. Soc., 94, 460-467, 1968.

Taylor, R. J., A new approach to the measurement of turbulent fluxes in the lower atmosphere, J. Fluid Mech., 10, 449-458, 1961.

Tillman, J. E., The indirect determination of stability, heat, and momentum fluxes in the atmospheric boundary layer from simple scalar variables during dry unstable conditions, J. Appl. Meteorol., 11, 783792, 1972.

Weaver, H. L., Temperature and humidity flux-variance relations determined by one-dimensional eddy correlation, Boundary Layer Meteorol., 53, 77-91, 1990.

Wesely, M. L., Use of variance techniques to measure dry air-surface exchange rates, Boundary Layer Meteorol., 44, 13-31, 1988.

Wyngaard, J. C., and O. R. Cote, The budgets of turbulent kinetic energy and temperature variance in the atmospheric surface layer, $J$. Atmos. Sci., 28, 190-201, 1971.

Wyngaard, J. C., O. R. Cote, and Y. Izumi, Local free convection, similarity and the budgets of shear stress and heat flux, J. Atmos. Sci., 28, 1171-1182, 1971.

Zilitinkevich, S. S., and D. V. Chalikov, Determining the universal wind-velocity and temperature profiles in the atmospheric boundary layer, Izv. Atmos. Oceanic Phys., 4, 294-302, 1968.

C-I Hsieh, G. G. Katul, K. R. Knoerr, and J. T. Sigmon, School of the Environment, Box 90328, Duke University, Durham, NC 277080328. (e-mail: gaby@acpub.duke.edu)

J. Schieldge, Jet Propulsion Laboratory, California Institute of Technology, Pasadena, CA 91109-8099.

(Received February 23, 1995; revised April 24, 1996; accepted April 26, 1996.) 